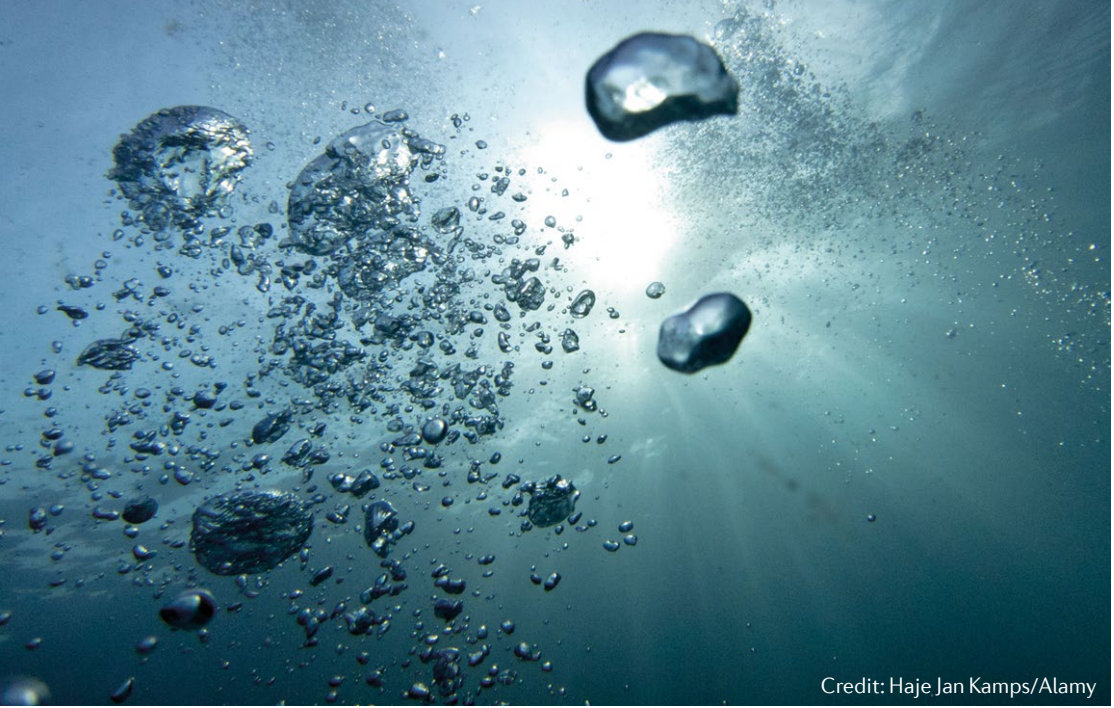

Estimating spleen size from abdominal magnetic resonance imaging (MRI) in a cohort of liver steatosis enabled direct genetic association testing for SNPs in the PDE10A gene region; one SNP, rs3008049, was indeed found to be significantly associated with spleen size.

Finally, the researchers determined the evolutionary history of selected SNPs. The PDE10A SNP rs3008049 had the highest frequency in Bajau but was also found in Saluans and Han-Chinese, suggesting that rs3008049 was evolutionarily selected as a standing variant.

The results of this study exemplify the co-evolution of cultural and genetic adaptations to extreme conditions and provide evidence for local genomic selection at PDE10A to improve hypoxia tolerance via lifestyle of the sea nomads.

Michelle Trenkmann, Associate Editor, Nature Communications.

ORIGINAL ARTICLE Ilardo, M. A. et al. Physiological and genetic adaptations to diving in sea nomads. Cell 173, 569-580 (2018) spleen size in response to the unique
Next, the team generated $K d m 6 b$ loss-of-function mutants using RNA interference (RNAi). Knockdown of $\mathrm{KDM} 6 \mathrm{~B}$ at $26^{\circ} \mathrm{C}$ led to male-to-female sex reversal. Compared with controls, decreased expression of the testicular Sertoli cell markers Amh and Sox9 and a concomitant increase in expression of the ovarian markers Cyp19a1 and Foxl2 after sex determination confirmed activation of the female development pathway.

Aiming to identify the target genes of KDM6B, the authors analysed six previously identified male-biased genes, two of which, Dmrt1 and Rbm20, exhibited mRNA levels that were reduced by more than half in KDM6Bdeficient mutants. Overexpressing Dmrt1 in KDM6B-deficient gonads at male-producing temperatures was sufficient to rescue the knockdown, with morphology and expression patterns resembling those of controls, leading to activation of the male pathway in close to $90 \%$ of cases.

The chromatin modifier KDM6B is responsible for the demethylation of trimethylated histone $\mathrm{H} 3$ lysine 27 (H3K27me3), a histone mark that is associated with repressed transcription

\section{Estimating heritability without genetic testing}

Heritability, the proportion of variation in a particular trait that is attributable to genetic factors, is key to predicting disease risk. A recent study reports the use of electronic health records (EHRs), which capture a range of clinical data, as a resource for studying the heritability of traits.

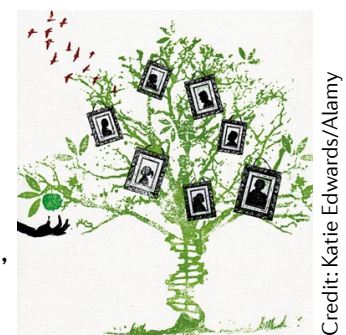

Given that heritability underlies the predictive nature of family history for a particular disease or trait, it has been traditionally studied in family cohort studies, in particular twin studies, which are often of limited sample size. The authors recognized the opportunity provided by EHRs as a large-scale source of familial relationship data. While EHRs contain no record of genetic relatedness between patients, they do contain emergency contacts, which the team used to map the relatedness within three hospital's patient populations.

The researchers obtained 3,550,598 inpatient EHRs used at the hospitals of Columbia University Medical Center, Weill Cornell Medical Center and Mount Sinai Health System, which provided 6,587,594 emergency contacts. Of these family members, 2,191,695 were patients themselves; $1,902,827$ of these provided an additional 1,588,134 emergency contacts who were next of kin. Using a newly developed algorithm for extracting relationships, called relationship inference from the electronic health record (RIFTEHR), the researchers could identify 7.4 million familial relationships across the three medical centres, without compromising patient privacy.

Clinical and genetic data were used to validate the relationships, with positive predictive values between $66 \%$ and $99 \%$ among first-degree relatives. For example, PLINK was used to estimate relatedness for 302 patients with RIFTEHR-predicted relationships from Columbia, who had consented to re-use of their genetic data. Of 172 predicted relationships, 134 were parent-offspring relationships, nearly all of which showed the expected genetic relatedness of $50 \%$.

Based on the more than 560,000 inferred pedigrees and using only medical data from the EHRs, Polubriaginof et al. then estimated the heritability of 500 traits adjusted for age and sex using sequential oligogenic linkage analysis routines (SOLAR). The resultant heritability estimates were similar across the three centres and matched those of previously published studies. The study also resolves an ongoing debate regarding the relative heritability of plasma levels of different cholesterol types, reporting that HDL cholesterol levels are more heritable than LDL cholesterol levels.

Taken together, this study showcases the utility of (accurate) EHRs for disease genetics, even without the genetics.
ORIGINAL ARTICLE Polubriaginof, F. C. G. et al. Disease heritability inferred from familial relationships reported in medical records. Cell https://doi.org/10.1016/j. cell.2018.04.032 (2018) 